Research Article

\title{
Control of Multiple Viruses Interacting and Propagating in Multilayer Networks
}

\author{
Xiao Tu $\mathbb{D},{ }^{1}$ Guo-Ping Jiang $\mathbb{D},{ }^{2}$ Yurong Song $\mathbb{D},{ }^{2}$ and Xiaoling Wang ${ }^{2}$ \\ ${ }^{1}$ School of Computer Science, Nanjing University of Posts and Telecommunications, Nanjing 210003, China \\ ${ }^{2}$ College of Automation, Nanjing University of Posts and Telecommunications, Nanjing 210003, China
}

Correspondence should be addressed to Guo-Ping Jiang; jianggp@njupt.edu.cn

Received 26 March 2020; Accepted 9 June 2020; Published 10 July 2020

Academic Editor: Bo Shen

Copyright $\odot 2020$ Xiao Tu et al. This is an open access article distributed under the Creative Commons Attribution License, which permits unrestricted use, distribution, and reproduction in any medium, provided the original work is properly cited.

Experimental studies involving control against virus propagation have attracted the interest of scientists. However, most accomplishments have been constrained by the simple assumption of a single virus in various networks, but this assumption apparently conflicts with recent developments in complex network theory, which details that each node might play multiple roles in different topological connections. Multiple viruses propagate through individuals via different routes, and thus, each individual component could be located in various positions of differing importance in each virus propagation process in each network. Therefore, we propose several control strategies for establishing a multiple-virus interaction and propagation model involving multiplex networks, including a novel Multiplex PageRank target control model and a multiplex random control model. Using computer experiments and simulations derived from actual examples, we exploit several actual cases to determine the relationship of the relative infection probability with the immunization probability. The results demonstrate the differences between our multiple-virus interaction and propagation model and the single-virus propagation model and verify the effectiveness of our novel Multiplex PageRank target control strategy. Moreover, we use parallel computing for simulating and identifying the relationships of the immunization thresholds with both interaction coefficients, which is beneficial for further practical applications because it can reduce the multiple interactions between viruses and allows achieving a greater effect through the immunization of fewer nodes in the multilayer networks.

\section{Introduction}

The structure and dynamics of multilayer networks have attracted much attention from scientific communities [1-24]. Comprised of a set of networks combined with interacting layers, these multilayer networks properly describe a variety of realistic complex systems, such as financial networks [18], ecological networks [19], information networks [20], and transportation networks [21].

Recently, many studies have attempted to discover the dynamics of viral propagation in multiplex networks. Along this line, various methods aiming at studying the virus propagation process in multilayer networks have been proposed and explored, and some examples include competing epidemics [25], the effect of the interconnected network structure [17], and the interaction of viruses and information [26]. More explicitly, several virus spreading models in partially overlapped networks have been proposed, and these networks are described as distinct networks that contain partially identical individuals [27-29]. By considering one virus spreading via multiple routes [6] or one virus spreading in multiple species [30], corresponding virus spreading models in multilayer networks have been proposed. Interestingly, these measures are not considerably affected by multiple viral interactions.

As mentioned above, although some accomplishments have been achieved by focusing on the effect of multilayer topology on virus dynamics and the resulting threshold, the influence of such improved complexity on control strategies remains unexplored [31-36]. In conventional research on virus control, the vaccinated candidate nodes are commonly chosen randomly or spontaneously according to their 
topological properties, such as degree, betweenness, $k$-shell, and PageRank [37-42]. Therefore, an interesting problem presents itself, which we aim to address in this work. If we consider the basic control cases in multilayer networks, how do they affect virus propagation?.

Here, using the multiple-virus spreading model in multilayer networks, we study the performance of some novel control strategies, including novel Multiplex PageRank targeted control and multiplex random control strategies, in multilayer networks. Based on the generating function theory, extensive computational simulations are performed to assess our measure in various cases, and we find that the effectiveness of the proposed control strategies relies on the interaction relationship between multiple viruses in multilayer networks.

\section{Multiple-Virus Interaction and Propagation}

Unlike the existing research on single-virus propagation in overlapping networks and double virus propagation in conventional networks, we focus on multiple viruses propagating through individuals, who are integrated into all layers of a multilayer network formed by different propagation routes. In our model, we briefly exploit a double-layer network containing network $A$ and network Bof the same size $N$ in which the nodes in both layers adopt one-to-one links. A schematic of the multilayer network is shown in Figure 1. The dashed lines represent the inner links of integrated individuals and their correspondence in the other layer. For the simulation, we construct each layer using a BA scale-free network model consisting of $N$ nodes; this network was primarily constructed based on a random network of $m_{0}$ nodes, and a new node with $m$ links to the existing nodes was then introduced until the network contained a total of $N$ nodes. In our model, we assume that the two viruses propagate according to their respective transmissibility in each layer, and the simulation is initiated by infecting several randomly chosen individuals in the multilayer network.

The propagation process follows a multiple-virus propagation model $\left(S_{A} I_{A} S_{A}-S_{B} I_{B} S_{B}\right)$ in a multilayer network, where each node can be divided into four compartments relevant to the two viruses: the individuals who are susceptible to both the virus in network $A$ and the virus in network $B\left(S_{A} S_{B}\right)$, the individuals who are susceptible to the virus propagated in $A$ while infected with the virus propagated in $B\left(S_{A} I_{B}\right)$, the individuals who are infected with the virus propagated in $A$ while susceptible to the virus propagated in $B\left(I_{A} S_{B}\right)$, and the individuals who are infected with both the virus propagated in $A$ and the virus propagated in $B\left(I_{A} I_{B}\right)$. An individual at state $S_{A}$ in $A$ can be converted to state $I_{A}$ if it comes in contact with any neighbor at state $I_{A}$, which occurs with the probability $\alpha_{A}^{S_{B}}$ if the individual is at state $S_{B}$ in $B$. An individual at state $S_{A}$ in $A$

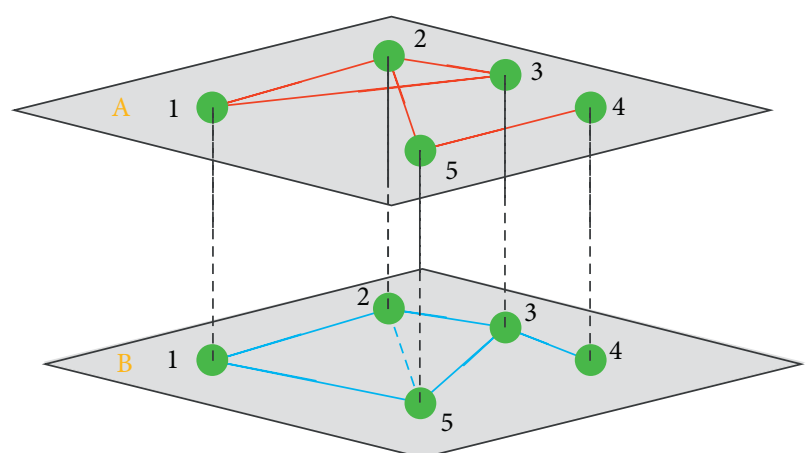

FIgURE 1: Multilayer network; the dashed lines show the inner links of the integrated nodes. Prior to the initiation of propagation, all individuals are in the susceptible state, which is represented by a green circle.

can be converted to state $I_{A}$ with the probability $\alpha_{A}^{I_{B}}$ if the individual is at state $I_{B}$ in $B$. Similar to $A$, an individual at state $S_{B}$ in $B$ can be converted to state $I_{B}$ with the probability $\alpha_{B}^{S_{A}}$ if the individual is at state $S_{A}$ in $A$. An individual at state $S_{B}$ in $B$ can be converted to $I_{B}$ state with the probability $\alpha_{B}^{I_{A}}$ if the individual is at state $I_{A}$ in $A$. An individual at state $I_{A}$ might be cured and returned to state $S_{A}$ with the probability $\mu_{A}$, whereas an individual at state $I_{B}$ might be cured and return to state $S_{B}$ with the probability $\mu_{B}$.

Moreover, $\alpha_{A}^{S_{B}}$ and $\alpha_{A}^{I_{B}}$ are related by the interaction coefficient $c_{A}^{I_{B}}$ such that $\alpha_{A}^{I_{B}}=c_{A}^{I_{B}} \alpha_{A}^{S_{B}}$, where $c_{A}^{I_{B}}$ indicates the influence of the virus in $B$ on the spreading probability of the virus in $A$, whereas $\alpha_{B}^{S_{A}}$ and $\alpha_{B}^{f_{A}}$ are related by the interaction coefficient $c_{B}^{I_{A}}$ such that $\alpha_{B}^{I_{A}}=c_{B}^{I_{A}} \alpha_{B}^{S_{A}}$, where $c_{B}^{I_{A}}$ refers to the influence of the virus in $A$ on the propagation probability of the virus in $B$. Notably, $c_{A}^{I_{B}} \geq 0, \alpha_{A}^{S_{B}} \in[0,1]$, and $\alpha_{A}^{I_{B}} \in[0,1]$. In particular, $c_{A}^{I_{B}}=0$ indicates that the virus in $B$ is immune to the virus in $A ; 0<c_{A}^{I_{B}}<1$ indicates that the virus in $B$ inhibits the virus in $A ; c_{A}^{I_{B}^{A}}=1$ indicates that the virus in $B$ has no effect on the virus in $A$; and $c_{A}^{I_{B}}>1$ indicates that the virus in $B$ enhances the virus in $A$. In addition, the spreading probability $\alpha_{A}^{I_{B}}$ must satisfy $\alpha_{A}^{I_{B}} \leq 1$; therefore, if $c_{A}^{I_{B}} \alpha_{A}^{S_{B}}>1$, then $\alpha_{A}^{I_{B}}=1$. The conditions in $B$ are similar to those in $A$.

Because we use $A$ and $B$ to denote the two virus propagation routes, $A_{j i}$ and $B_{j i}$ are denoted as the edges from individual $j$ to individual $i$ by the routes of each propagated virus, which are equal to 1 if edges exist and 0 otherwise. We adopt the process of a continuous time Markov chain to describe the interaction of multiple propagating viruses by defining $X_{i}^{t}$ and $Y_{i}^{t}$ as measures of the random variables $X$ and $Y$ of node $i$ at time $t$, respectively. For each $i \in\{1,2, \ldots, N\}$, the random variables $X_{i}$ and $Y_{i}$ satisfy $X_{i}: \quad\left\{S_{A}, I_{A}\right\} \longrightarrow\{0,1\}$ and $Y_{i}$ : $\left\{S_{B}, I_{B}\right\} \longrightarrow\{0,1\}$. Therefore, the continuous time Markov chain equation of the node state evolution over time is represented as follows: 


$$
\begin{aligned}
& \operatorname{Pr}\left[X_{i}^{t+1}=1, Y_{i}^{t+1}=0 \mid X_{i}^{t}=0, Y_{i}^{t}=0\right]=\alpha_{A}^{S_{B}} \Delta t \sum_{j \in N_{i}} 1_{\left\{X_{j}^{t}=1\right\}}+o(\Delta t), \\
& \operatorname{Pr}\left[X_{i}^{t+1}=1, Y_{i}^{t+1}=1 \mid X_{i}^{t}=0, Y_{i}^{t}=1\right]=\alpha_{A}^{I_{B}} \Delta t \sum_{j \in N_{i}} 1_{\left\{X_{j}^{t}=1\right\}}+o(\Delta t), \\
& \operatorname{Pr}\left[X_{i}^{t+1}=0, Y_{i}^{t+1}=0 \mid X_{i}^{t}=1, Y_{i}^{t}=0\right]=\mu_{A} \Delta t+o(\Delta t), \\
& \operatorname{Pr}\left[X_{i}^{t+1}=0, Y_{i}^{t+1}=1 \mid X_{i}^{t}=0, Y_{i}^{t}=0\right]=\alpha_{B}^{S_{A}} \Delta t \sum_{j \in N_{i}} 1_{\left\{Y_{j}^{t}=0\right\}}+o(\Delta t), \\
& \operatorname{Pr}\left[X_{i}^{t+1}=1, Y_{i}^{t+1}=1 \mid X_{i}^{t}=1, Y_{i}^{t}=0\right]=\alpha_{B}^{I_{A}} \Delta t \sum_{j \in N_{i}} 1_{\left\{Y_{j}^{t}=1\right\}}+o(\Delta t), \\
& \operatorname{Pr}\left[X_{i}^{t+1}=0, Y_{i}^{t+1}=0 \mid X_{i}^{t}=0, Y_{i}^{t}=1\right]=\mu_{B} \Delta t+o(\Delta t) .
\end{aligned}
$$

where $\operatorname{Pr}$ is the probability and $\Delta t>0$ is a time step, $1_{\{\chi\}}$, which is equal to 1 if $\chi$ is true or 0 otherwise. If $\lim _{\Delta t \rightarrow 0}(f(\Delta t) / \Delta t)=0, f(\Delta t)$ can be written as $o(\Delta t)$. In particular, $1_{\left\{X_{j}^{t}=1\right\}}$ can be represented by $\operatorname{Pr}\left[X_{j}=1\right]$. Thus, the continuous Markov chain equation (1) can be transformed to a new random stochastic equation:

$$
\begin{aligned}
& \operatorname{Pr}\left[X_{i}^{t+1}=1, Y_{i}^{t+1}=0 \mid X_{i}^{t}=0, Y_{i}^{t}=0\right]=\alpha_{A}^{S_{B}} \Delta t \sum_{j \in N_{i}} \operatorname{Pr}\left[X_{j}^{t}=1\right]+o(\Delta t), \\
& \operatorname{Pr}\left[X_{i}^{t+1}=1, Y_{i}^{t+1}=1 \mid X_{i}^{t}=0, Y_{i}^{t}=1\right]=\alpha_{A}^{I_{B}} \Delta t \sum_{j \in N_{i}} \operatorname{Pr}\left[X_{j}^{t}=1\right]+o(\Delta t), \\
& \operatorname{Pr}\left[X_{i}^{t+1}=0, Y_{i}^{t+1}=0 \mid X_{i}^{t}=1, Y_{i}^{t}=0\right]=\mu_{A} \Delta t+o(\Delta t), \\
& \operatorname{Pr}\left[X_{i}^{t+1}=0, Y_{i}^{t+1}=1 \mid X_{i}^{t}=0, Y_{i}^{t}=0\right]=\alpha_{B}^{S_{A}} \Delta t \sum_{j \in N_{i}} \operatorname{Pr}\left[Y_{j}^{t}=1\right]+o(\Delta t), \\
& \operatorname{Pr}\left[X_{i}^{t+1}=1, Y_{i}^{t+1}=1 \mid X_{i}^{t}=1, Y_{i}^{t}=0\right]=\alpha_{B}^{I_{A}} \Delta t \sum_{j \in N_{i}} \operatorname{Pr}\left[Y_{j}^{t}=1\right]+o(\Delta t), \\
& \operatorname{Pr}\left[X_{i}^{t+1}=0, Y_{i}^{t+1}=0 \mid X_{i}^{t}=0, Y_{i}^{t}=1\right]=\mu_{B} \Delta t+o(\Delta t) .
\end{aligned}
$$

The value of the nodes reflects the average probability of the four states at time $t$, which are, respectively, denoted by $p_{i}^{S_{A} S_{B}}(t), p_{i}^{S_{A} I_{B}}(t), p_{i}^{I_{A} S_{B}}(t)$, and $p_{i}^{I_{A} I_{B}}(t)$ and satisfy

$$
p_{i}^{S_{A} S_{B}}(t)+p_{i}^{S_{A} I_{B}}(t)+p_{i}^{I_{A} S_{B}}(t)+p_{i}^{I_{A} I_{B}}(t)=1 .
$$

Thus, the average probability of the virus in $A$ at time $t$ is represented as $p_{i}^{I_{A}}(t)=p_{i}^{I_{A} S_{B}}(t)+p_{i}^{I_{A} I_{B}}(t)$ and that of the virus in $B$ at time $t$ is represented as $p_{i}^{I_{B}}(t)=p_{i}^{S_{A} I_{B}}(t)+p_{i}^{I_{A} I_{B}}(t)$. We defined the new state as $Z_{i}\left(\in\left[p_{i}^{S_{A} S_{B}}, p_{i}^{S_{A} I_{B}}, p_{i}^{I_{A} S_{B}}, p_{i}^{I_{A} I_{B}}\right]\right)$. According to the correlation theorem and the properties of the continuous time Markov chain, the transfer matrix $Q$ of the stochastic process can be obtained as follows:

$$
Q=\left[\begin{array}{cccc}
-\alpha_{A}^{S_{B}} \sum_{j} A_{j i} p_{j}^{I_{A}}-\alpha_{B}^{S_{A}} \sum_{j} B_{j i} p_{j}^{I_{B}} & -\alpha_{A}^{I_{B}} \sum_{j} A_{j i} p_{j}^{I_{A}}+\mu_{B} & \mu_{A}-\alpha_{B}^{S_{A}} \sum_{j} B_{j i} p_{j}^{I_{B}} & \mu_{A}+\mu_{B} \\
-\alpha_{A}^{S_{B}} \sum_{j} A_{j i} p_{j}^{I_{A}}+\alpha_{B}^{S_{A}} \sum_{j} B_{j i} p_{j}^{I_{B}} & -\alpha_{A}^{I_{B}} \sum_{j} A_{j i} p_{j}^{I_{A}}-\mu_{B} & \mu_{A}+\alpha_{B}^{S_{A}} \sum_{j} B_{j i} p_{j}^{I_{B}} & \mu_{A}-\mu_{B} \\
\alpha_{A}^{S_{B}} \sum_{j} A_{j i} p_{j}^{I_{A}}-\alpha_{B}^{S_{A}} \sum_{j} B_{j i} p_{j}^{I_{B}} & \alpha_{A}^{I_{B}} \sum_{j} A_{j i} p_{j}^{I_{A}}+\mu_{B} & -\mu_{A}-\alpha_{B}^{S_{A}} \sum_{j} B_{j i} p_{j}^{I_{B}} & -\mu_{A}+\mu_{B} \\
\alpha_{A}^{S_{B}} \sum_{j} A_{j i} p_{j}^{I_{A}}+\alpha_{B}^{S_{A}} \sum_{j} B_{j i} p_{j}^{I_{B}} & \alpha_{A}^{I_{B}} \sum_{j} A_{j i} p_{j}^{I_{A}}-\mu_{B} & -\mu_{A}+\alpha_{B}^{S_{A}} \sum_{j} B_{j i} p_{j}^{I_{B}}-\mu_{A}-\mu_{B}
\end{array}\right]
$$

Therefore, the Kolmogorov forward differential equations of the stochastic process (2) can be represented as follows:

$$
\dot{Z}_{i}=Z_{i} Q, \quad i \in\{1,2, \ldots, N\}
$$

We can expand the differential equations of node $i$ to obtain 


$$
\begin{aligned}
& \dot{p}_{S_{A} S_{B}}=\left(-\alpha_{A}^{S_{B}} \sum_{j} A_{j i} p_{j}^{I_{A}}-\alpha_{B}^{S_{A}} \sum_{j} B_{j i} p_{j}^{I_{B}}\right) p_{i}^{S_{A} S_{B}}+\left(-\alpha_{A}^{I_{B}} \sum_{j} A_{j i} p_{j}^{I_{A}}+\mu_{B}\right) p_{i}^{S_{A} I_{B}} \\
& +\left(\mu_{A}-\alpha_{B}^{S_{A}} \sum_{j} B_{j i} p_{j}^{I_{B}}\right) p_{i}^{I_{A} S_{B}}+\left(\mu_{A}+\mu_{B}\right) p_{i}^{I_{A} I_{B}} \\
& \dot{p}_{S_{A} I_{B}}=\left(-\alpha_{A}^{S_{B}} \sum_{j} A_{j i} p_{j}^{I_{A}}+\alpha_{B}^{S_{A}} \sum_{j} B_{j i} p_{j}^{I_{B}}\right) p_{i}^{S_{A} S_{B}}+\left(-\alpha_{A}^{I_{B}} \sum_{j} A_{j i} p_{j}^{I_{A}}-\mu_{B}\right) p_{i}^{S_{A} I_{B}} \\
& +\left(\mu_{A}+\alpha_{B}^{S_{A}} \sum_{j} B_{j i} p_{j}^{I_{B}}\right) p_{i}^{I_{A} S_{B}}+\left(\mu_{A}-\mu_{B}\right) p_{i}^{I_{A} I_{B}}, \\
& \dot{p}_{I_{A} S_{B}}=\left(\alpha_{A}^{S_{B}} \sum_{j} A_{j i} p_{j}^{I_{A}}-\alpha_{B}^{S_{A}} \sum_{j} B_{j i} p_{j}^{I_{B}}\right) p_{i}^{S_{A} S_{B}}+\left(\alpha_{A}^{I_{B}} \sum_{j} A_{j i} p_{j}^{I_{A}}+\mu_{B}\right) p_{i}^{S_{A} I_{B}} \\
& +\left(-\mu_{A}-\alpha_{B}^{S_{A}} \sum_{j} B_{j i} p_{j}^{I_{B}}\right) p_{i}^{I_{A} S_{B}}+\left(-\mu_{A}+\mu_{B}\right) p_{i}^{I_{A} I_{B}}, \\
& \dot{p}_{I_{A} I_{B}}=\left(\alpha_{A}^{S_{B}} \sum_{j} A_{j i} p_{j}^{I_{A}}+\alpha_{B}^{S_{A}} \sum_{j} B_{j i} p_{j}^{I_{B}}\right) p_{i}^{S_{A} S_{B}}+\left(\alpha_{A}^{I_{B}} \sum_{j} A_{j i} p_{j}^{I_{A}}-\mu_{B}\right) p_{i}^{S_{A} I_{B}} \\
& +\left(-\mu_{A}+\alpha_{B}^{S_{A}} \sum_{j} B_{j i} p_{j}^{I_{B}}\right) p_{i}^{I_{A} S_{B}}+\left(-\mu_{A}-\mu_{B}\right) p_{i}^{I_{A} I_{B}} .
\end{aligned}
$$

\section{Multiplex Control Strategy}

In contrast to the control of each noninteracted virus in each conventional network individually, the multiplex control strategy aims to immunize fewer nodes but achieve a greater effect on both of the interacting viruses in the multilayer network simultaneously. Thus, we compare our novel Multiplex PageRank target control with multiplex random control in a multilayer network.

Recently, researchers have focused on measuring the centrality of multiplex networks. The eigenvector multiplex centrality hypothesizes that the centrality of a node in one layer is impacted by its centrality in other layers by an overlapped influence matrix [43]. The versatility of nodes highlights the relevance of related nodes in different layers and applies to multilayer networks in which the corresponding nodes in different layers are connected by interlinks [44]. The Multiplex PageRank centrality utilizes the correlations among the degrees of nodes in different layers by means of a random walk subject to teleportation [45-49].

Among these centrality measures, the versatility of nodes is the only measure that considers the interlinks, whereas both the Multiplex PageRank centrality and the eigenvector multiplex centrality stipulate one-to-one links among the nodes in different layers, which are denoted inner links throughout the rest of the manuscript paper. An inner link induces a coupling relationship that will impact the distribution of centrality inside the same node. The major challenge when identifying the centrality of a node in a multiplex network with inner links is that the centrality depends on the relationship among the distinct types of links, which are also known as traditional links, between different nodes in the same layer. This study attempts to address the issue of the centrality distribution through a generalization of PageRank by considering the coupling relationship.

Our novel Multiplex PageRank measure is derived from a random population migration in an urban multilayer transport network, including a flight network $A$ and a railway network $B$, to obtain the rankings of the hub cities in terms of transportation. Because we aim to obtain the rankings of the hub cities in terms of transportation, two or more train stations or airports in a same city are considered single stations or airports. The connections between train stations or airports in different cities are identified by the existence of railways or airlines. The population is migrating according to the distribution coefficients $\beta_{A}$ and $\beta_{B}$, which satisfy $0 \leq \beta_{A}+\beta_{B} \leq 1$. This relationship indicates that some of the population $\left(\beta_{A}\right)$ prefer travelling by aircraft, some of the population $\left(\beta_{B}\right)$ prefer travelling by train, and some of the population $\left(1-\beta_{A}-\beta_{B}\right)$ refuse to travel because they are choosing to remain in cities due to personal interests. The total population of city $i$ at time $t$ is defined as $S_{i}(t)$. Therefore, we can obtain $S_{i}(t+1)$ as

$$
S_{i}(t+1)=\sum_{j} A_{j i} \frac{\beta_{A} S_{j}(t)}{H_{j}^{(A)}}+\sum_{j} B_{j i} \frac{\beta_{B} S_{j}(t)}{H_{j}^{(B)}}+\left(1-\beta_{A}-\beta_{B}\right) v_{i},
$$

where $H_{j}^{(A)}=\sum_{r} A_{j r}+\delta\left(0, \sum_{r} A_{j r}\right), H_{j}^{(B)}=\sum_{r} B_{j r}+\delta\left(0, \sum_{r} B_{j r}\right)$, and $\delta(x, y)$ is the Kronecker delta, which equals one if $x=y$ and zero if $x \neq y$ and can be exploited to control city $j$ without any transportation to other cities to keep the population of $j$ in itself, and $v_{i}$ is the personalized interests of 
the population staying in city $i$. By initializing each $S_{i}(0)$ by the value $N^{-1}$, as determined by the number of cities and repeating the migration process, we can obtain the rankings of the hub cities in terms of transportation and derive the rankings in terms of node importance in a multilayer network.

Based on our urban multiplex transport network, which has two layers, the generated Multiplex PageRank centrality measure is applied to a multiplex network of $M$ layers with $N$ nodes in each layer as follows:

$$
S_{i}(t+1)=\sum_{L} \sum_{j} g_{j i}^{(L)} \frac{\alpha_{L} S_{i}(t)}{H_{j}^{(L)}}+\left(1-\sum_{L} \alpha_{L}\right) v_{i},
$$

where $H_{j}^{(L)}=\sum_{r} g_{j r}^{(L)}+\delta\left(0, \sum_{r} g_{j r}^{(L)}\right)$,

$$
\begin{aligned}
& \dot{p}_{S_{A} S_{B}}=\left(-\alpha_{A}^{S_{B}} \sum_{j} A_{j i} p_{j}^{I_{A}}-\alpha_{B}^{S_{A}} \sum_{j} B_{j i} p_{j}^{I_{B}}\right) p_{i}^{S_{A} S_{B}}\left(1-w_{i}\right)+\left(-\alpha_{A}^{I_{B}} \sum_{j} A_{j i} p_{j}^{I_{A}}+\mu_{B}\right) p_{i}^{S_{A} I_{B}}\left(1-w_{i}\right) \\
& +\left(\mu_{A}-\alpha_{B}^{S_{A}} \sum_{j} B_{j i} p_{j}^{I_{B}}\right) p_{i}^{I_{A} S_{B}}\left(1-w_{i}\right)+\left(\mu_{A}+\mu_{B}\right) p_{i}^{I_{A} I_{B}}\left(1-w_{i}\right), \\
& \dot{p}_{S_{A} I_{B}}=\left(-\alpha_{A}^{S_{B}} \sum_{j} A_{j i} p_{j}^{I_{A}}+\alpha_{B}^{S_{A}} \sum_{j} B_{j i} p_{j}^{I_{B}}\right) p_{i}^{S_{A} S_{B}}\left(1-w_{i}\right)+\left(-\alpha_{A}^{I_{B}} \sum_{j} A_{j i} p_{j}^{I_{A}}-\mu_{B}\right) p_{i}^{S_{A} I_{B}}\left(1-w_{i}\right) \\
& +\left(\mu_{A}+\alpha_{B}^{S_{A}} \sum_{j} B_{j i} p_{j}^{I_{B}}\right) p_{i}^{I_{A} S_{B}}\left(1-w_{i}\right)+\left(\mu_{A}-\mu_{B}\right) p_{i}^{I_{A} I_{B}}\left(1-w_{i}\right), \\
& \dot{p}_{I_{A} S_{B}}=\left(\alpha_{A}^{S_{B}} \sum_{j} A_{j i} p_{j}^{I_{A}}-\alpha_{B}^{S_{A}} \sum_{j} B_{j i} p_{j}^{I_{B}}\right) p_{i}^{S_{A} S_{B}}\left(1-w_{i}\right)+\left(\alpha_{A}^{I_{B}} \sum_{j} A_{j i} p_{j}^{I_{A}}+\mu_{B}\right) p_{i}^{S_{A} I_{B}}\left(1-w_{i}\right) \\
& +\left(-\mu_{A}-\alpha_{B}^{S_{A}} \sum_{j} B_{j i} p_{j}^{I_{B}}\right) p_{i}^{I_{A} S_{B}}\left(1-w_{i}\right)+\left(-\mu_{A}+\mu_{B}\right) p_{i}^{I_{A} I_{B}}\left(1-w_{i}\right), \\
& \dot{p}_{I_{A} I_{B}}=\left(\alpha_{A}^{S_{B}} \sum_{j} A_{j i} p_{j}^{I_{A}}+\alpha_{B}^{S_{A}} \sum_{j} B_{j i} p_{j}^{I_{B}}\right) p_{i}^{S_{A} S_{B}}\left(1-w_{i}\right)+\left(\alpha_{A}^{I_{B}} \sum_{j} A_{j i} p_{j}^{I_{A}}-\mu_{B}\right) p_{i}^{S_{A} I_{B}}\left(1-w_{i}\right) \\
& +\left(-\mu_{A}+\alpha_{B}^{S_{A}} \sum_{j} B_{j i} p_{j}^{I_{B}}\right) p_{i}^{I_{A} S_{B}}\left(1-w_{i}\right)+\left(-\mu_{A}-\mu_{B}\right) p_{i}^{I_{A} I_{B}}\left(1-w_{i}\right) .
\end{aligned}
$$

We start the multiplex target control strategy by immunizing the top $\phi \in[0,1]$ ranked individuals in the multilayer network or the multiplex random control strategy by immunizing an arbitrary number of $\phi$ individuals in the multilayer network before virus propagation begins. An immunized individual is aware of, takes preventive action against, and is immunized to both viruses in both layers and thus cannot be infected or is infectious throughout the propagation process. We define the immunization state of an individual $i$ as $w_{i}$, which is equal to 1 if $i$ is immunized and to 0 otherwise.

After immunization, we propagate both viruses in the multilayer network, and this process is initiated by infecting several random susceptible nonimmunized individuals in each layer. We then obtain expansion of the Kolmogorov forward differential equations of the stochastic process of the continuous time Markov Chains for the multiple-virus propagation model, which is constrained by the multiplex control strategy for each node $i$ as represented in (9).

\section{Simulation}

Considering the interaction relationships between the two viruses, several actual cases are investigated through computer experiments and simulations. By adopting $\rho_{\phi}$ as the infection probability at an immunization probability $\phi$ at the steady state and $\rho_{0}$ as the infection probability without control at the steady state, we can obtain the immunized probability versus the steady infection probability for the following. The common parameters are set to $N=10000$, $\alpha_{A}^{S_{B}}=0.2, \alpha_{B}^{S_{A}}=0.2, \mu_{A}=0.8$, and $\mu_{B}=0.8$, and $\phi$ ranges from 0 to 1 at $1 \%$ intervals. Moreover, we adopt $m_{0}=20$ and $m=8$ to construct network $A$, and $m_{0}=30$ and $m=12$ are used to construct network $B$. Moreover, $I_{A}$ means the nodes infected with the virus in network $A, I_{B}$ means the nodes infected with the virus in network $B$, and $I_{A} \cup I_{B}$ means the union of $I_{A}$ and $I_{B}$, i.e., all infected nodes without considering the types of viruses. The control results are averaged by simulating 100 time steps 1000 times in 50 multilayer networks. 
Because an individual who is affected with whooping cough or measles will infect another individual [50], we adopt $c_{A}^{I_{B}}=0$ for whopping cough or $c_{B}^{I_{A}}=0$ for measles. Because there are more propagation routes for measles than whooping cough, we adopt $A$ to denote the propagation routes of whooping cough and $B$ to denote the propagation routes of measles. By deciding to mainly immunize against measles and to immunize against whooping cough indirectly, we adopt the distribution coefficients $\beta_{A}=0.35$ and $\beta_{B}=0.5$ because measles is more dangerous than whooping cough. The simulation results are shown in Figure 2. As shown in Figure 2(a), the immunization threshold of the multiplex random control of whooping cough is in a high value about 0.8 , and the decreasing rate of the curve is smaller and smaller, whereas the immunization threshold of the multiplex target control of whooping cough is constrained to a very low value, which is affected by the control of measles with our novel Multiplex PageRank method and the limitations caused by the existence of fewer propagation routes for whooping cough compared with those of multiplex random control. In addition, as shown in Figure 2(b), the immunization threshold of the multiplex random control of whooping cough is about equal to that of measles, but the decreasing rate of the curve is constant, whereas a turning point can be found in the curve of the multiplex target control of measles before the immunization threshold is reached. This finding indicates that measles benefits from a greater number of propagation routes, and thus, the infection probability of our Multiplex PageRank target control decreases at a lower rate compared with that observed with multiplex random control. The turning point occurs at the value of approximately 0.12 when the population has been completely immunized against whooping cough, and thus, the control of the population to whooping cough results in a decrease in the immunization rate of measles. Figure 2(c) presents the union of infections of whooping cough and measles in the multilayer network. The decreasing infection probability rate remains at a steady rate. As shown by the results, the Multiplex PageRank target control strategy immunizes fewer nodes and has a greater effect on both viruses simultaneously in the multilayer network.

A computer that is affected by countermeasures, which are also classified as infectious malware in China, can avoid infection by some computer viruses [51]. In addition, a computer affected by these computer viruses is not affected by the propagation of countermeasures; thus, we adopt $c_{A}^{I_{B}}=$ 1 for countermeasures and $c_{B}^{I_{A}}=0$ for computer viruses. Because the propagation routes of computer viruses are increased compared with those of countermeasures, we adopt $A$ to denote the propagation routes of countermeasures and $B$ to denote the propagation routes of computer viruses. By deciding to mainly immunize against computer viruses and to immunize against countermeasures indirectly, we adopt the distribution coefficients $\beta_{A}=0.35$ and $\beta_{B}=0.5$ because computer viruses are more dangerous than countermeasures. The simulation results are shown in Figure 3. The results shown in Figure 3(a) demonstrate that the immunization threshold of the multiplex target control of the countermeasures is constrained to a lower value than that of multiplex random control. Even if we mainly concentrate on immunizing against computer viruses, the propagation of countermeasures is constrained by the decreased propagation routes. However, the immunization threshold of the multiplex random control of the countermeasures is at a high value about 0.8 , and the decreasing rate of the curve is constant. In addition, Figure 3(b) shows the existence of several turning points in the curve of multiplex target control against computer viruses prior to attaining the immunization threshold. However, the immunization threshold of the multiplex random control of computer viruses is in a high value about 0.8 , and the decreasing rate of the curve is constant. This finding indicates that the computer viruses are first immunized by the countermeasures. The first turning point is found at a value of approximately 0.2 when the countermeasures propagate within the topranked nodes are mostly immunized, which results in an increase in the probability of infection with computer viruses. However, the infection probability soon begins to decrease at a value of approximately 0.27 after the population is fully immunized against countermeasures, which also immunizes against computer viruses. Figure 3(c) presents the union of the infections by countermeasures and computer viruses in the multilayer network. The decreasing rate of the infection probability remains unchanged, and the results show that our Multiplex PageRank target control strategy involves the control of fewer nodes to achieve greater effects on both viruses simultaneously in the multilayer network.2

Assuming that an individual who is affected with hepatitis $C$ or $B$ virus will show inhibition against the other virus and that the inhibition of hepatitis $B$ virus by hepatitis $C$ virus is stronger than that of hepatitis $C$ virus by hepatitis $B$ virus [52], we adopt $c_{A}^{I_{B}}=0.5$ for hepatitis $C$ virus and $c_{B}^{I_{A}}=$ 0.3 for hepatitis $B$ virus. Because there are more propagation routes for hepatitis $B$ virus than for hepatitis $C$ virus, we adopt $A$ to denote the propagation routes of hepatitis $C$ virus and $B$ to denote the propagation routes of hepatitis $C$ virus. Based on the decision to mainly immunize against hepatitis $B$ virus and sometimes immunize against hepatitis $C$ virus, we adopt the distribution coefficients $\beta_{A}=0.35$ and $\beta_{B}=0.5$ because hepatitis $B$ virus is more dangerous than hepatitis $C$ virus. The simulation results are shown in Figure 4. As shown in Figure 4(a), the immunization threshold of the multiplex random control of hepatitis $C$ virus is at a high value about 0.8 , and the decreasing rate of the curve is constant, whereas the immunization threshold of the multiplex target control of the hepatitis $\mathrm{C}$ virus is constrained to a lower value based on control with our novel Multiplex PageRank, the suppression of hepatitis B virus, and the limitation of the propagation routes of hepatitis $C$ virus compared with multiplex random control. Moreover, the results in Figure 4(b) demonstrate a change in the decreasing rate of the infection probability obtained with multiplex target control of hepatitis B virus before attaining the immunization threshold. However, the immunization threshold of the multiplex random control of hepatitis $B$ virus is in a high value about 0.8 , and the decreasing rate of the curve is constant. These results indicate that hepatitis B 


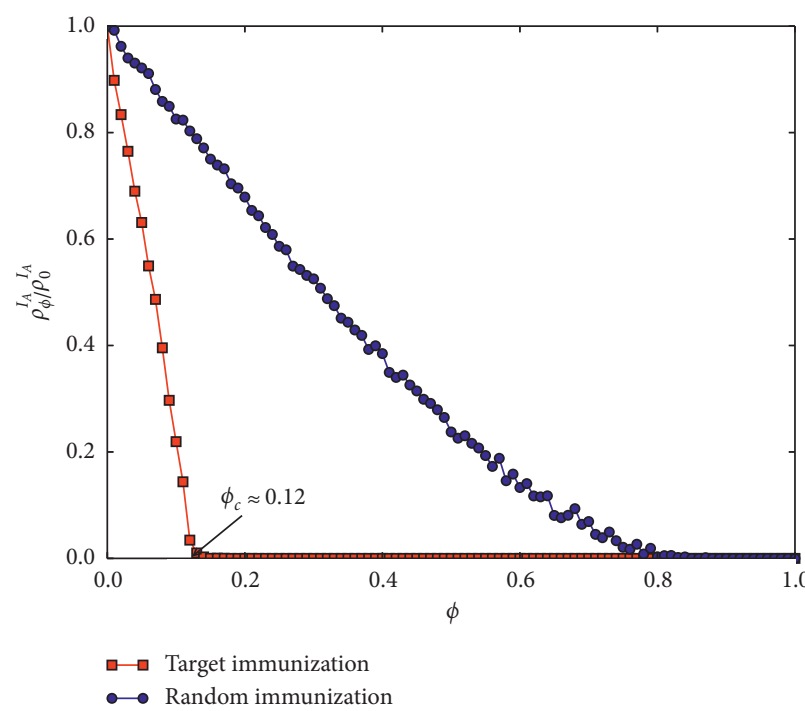

(a)

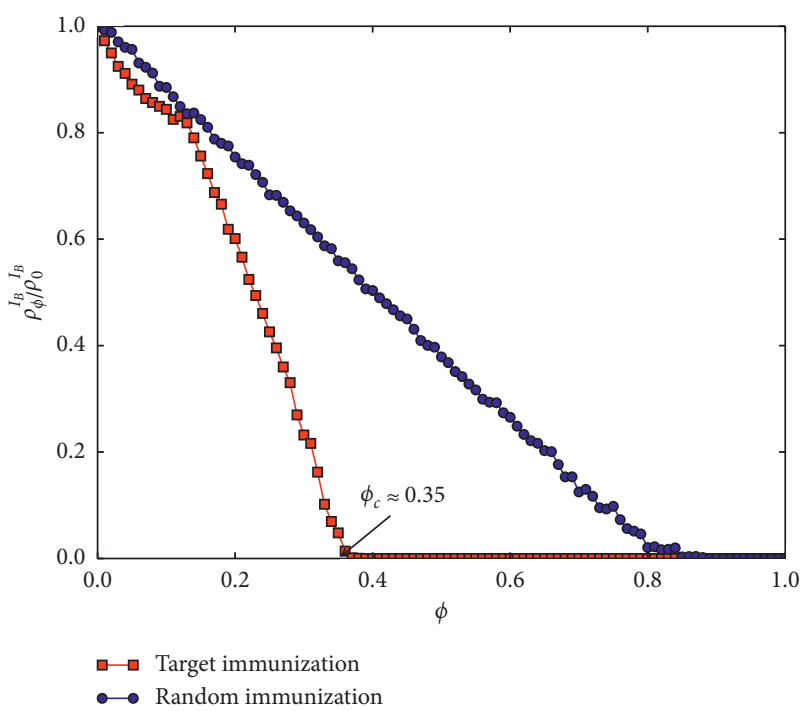

(b)

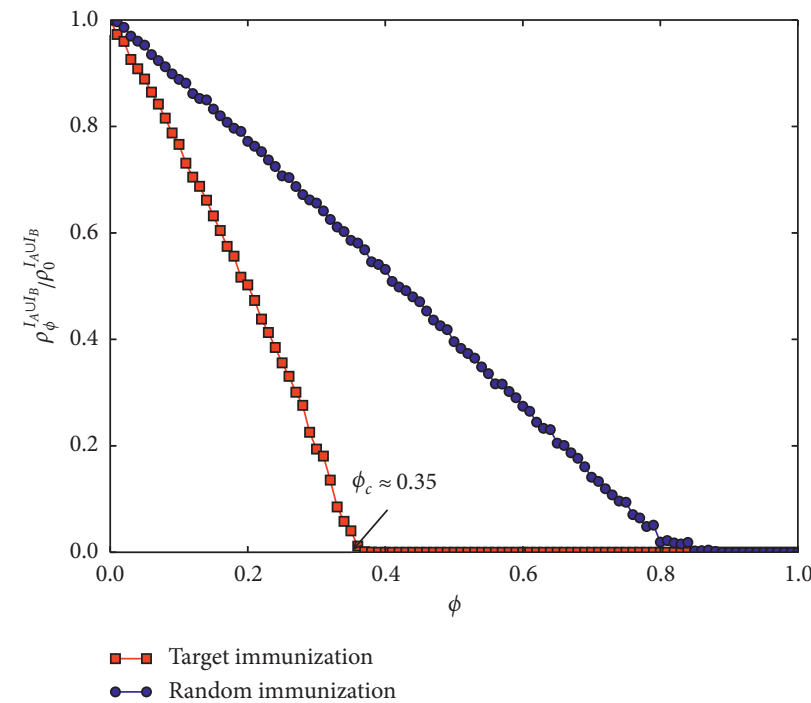

(c)

Figure 2: Relative size $\left(\rho_{\phi} / \rho_{0}\right)$ of infected clusters versus the immunization probability $\phi$ for multiple targets or random control in a double layer network that involves whooping cough and measles: (a) whooping cough-infected clusters, (b) measles-infected clusters, and (c) clusters infected with both whooping cough and measles. The arrow denotes the immunization threshold $\phi_{c}$ of our novel Multiplex PageRank target control.

virus is first suppressed by hepatitis $C$ virus. The change in the decreasing rate of the infection is probability at a value of approximately 0.24 occurred when the population is completely immunized against hepatitis C virus, which occasioning results in a decline in the decreasing rate of the infection probability of hepatitis B virus. However, the decreasing rate of the infection probability soon increases with an increase in the immunization probability. Figure 4(c) presents the union of infection with hepatitis C and $B$ viruses in the multilayer network. The decreasing rate of the infection probability is maintained at a constant rate. Our Multiplex PageRank target control strategy indicates that the control of fewer nodes can achieve a greater effect on both viruses simultaneously in the multilayer network.
Because tuberculosis does not affect HIV but an individual who is infected with HIV will be more susceptible to infection by tuberculosis [53], we adopt $c_{A}^{I_{B}}=1.5$ for tuberculosis and $c_{B}^{I_{A}}=1$ for HIV. Due to the higher number of propagation routes for HIV compared with tuberculosis, we adopt $A$ to denote the propagation routes of tuberculosis and $B$ to denote the propagation routes of HIV. By deciding to mainly immunize against HIV and only slightly immunize against tuberculosis, we adopt the distribution coefficients $\beta_{A}=0.35$ and $\beta_{B}=0.5$ because HIV is more dangerous than tuberculosis. The simulation results are shown in Figure 5. The results in Figure 5(a) reveal a small change in the decreasing rate of the infection probability with multiplex target control of tuberculosis before attaining the 


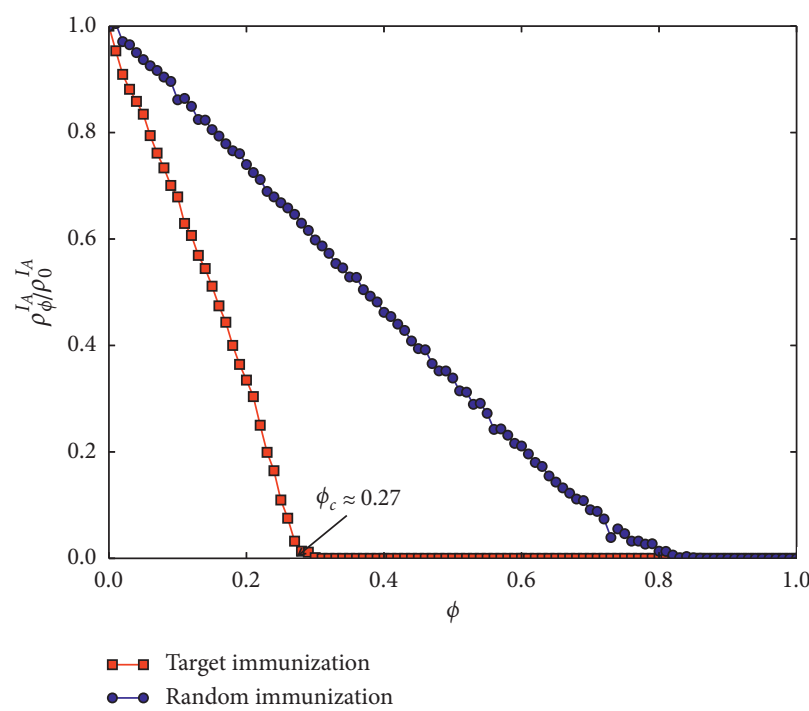

(a)

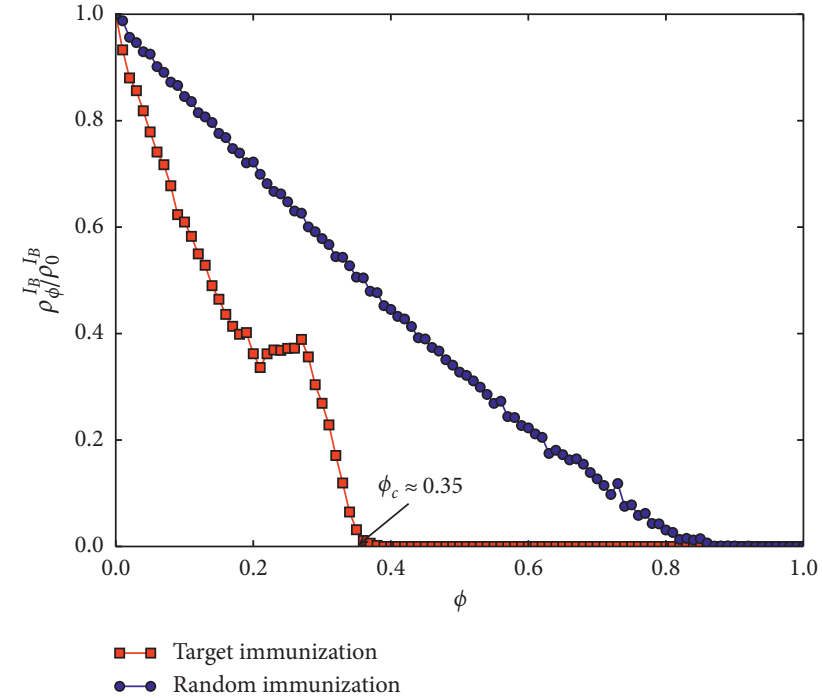

(b)

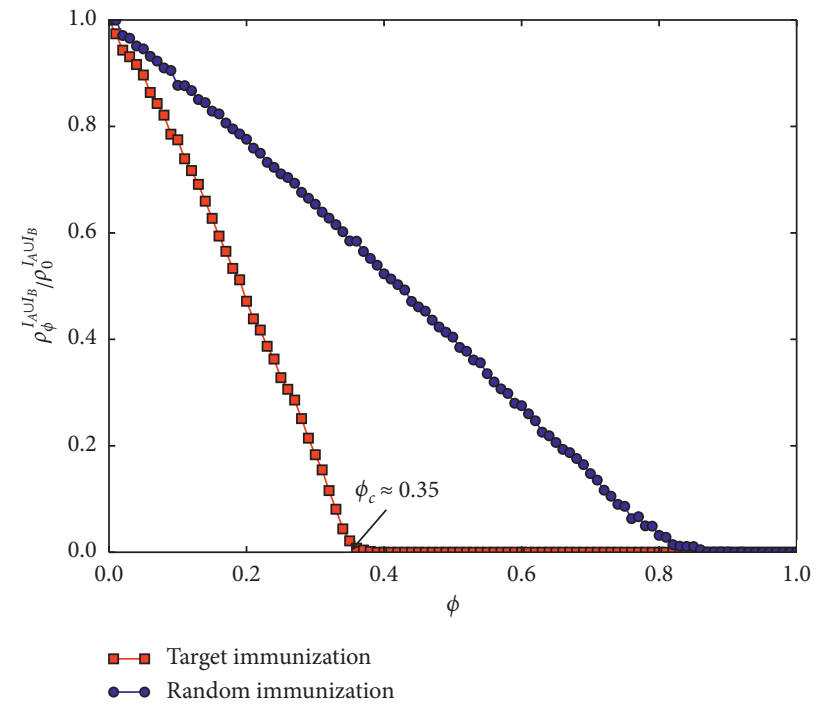

(c)

FiguRE 3: Relative size $\left(\rho_{\phi} / \rho_{0}\right)$ of infected clusters versus the immunization probability $\phi$ for multiplex target or random control in a doublelayer network that includes countermeasures and computer viruses: (a) countermeasures-infected clusters, (b) computer virus-infected clusters, and (c) clusters infected with both countermeasures and computer viruses. The arrow denotes the immunization threshold $\phi_{c}$ of our novel Multiplex PageRank target control.

immunization threshold. However, the immunization threshold of the multiplex random control of tuberculosis is at a high value about 0.8 , and the decreasing rate of the curve is constant. This finding indicates that tuberculosis is first enhanced by HIV. The change in the decreasing rate of the infection probability occurs at the value of approximately 0.22 when HIV is propagated in the top ranking nodes, which are mostly immunized, and this finding results in a small increase in the decreasing rate of the infection probability of tuberculosis. However, even if tuberculosis infection has been enhanced, it is constrained by its fewer propagation routes; thus, the population is completely immunized against tuberculosis before it is immunized against HIV. Moreover, as shown in Figure 5(b), the immunization threshold of the multiplex random control of HIV is at a high value about 0.8 , and the decreasing rate of the curve is constant. We also find a small change in the rate of the infection probability obtained through multiplex target control for HIV before the immunization threshold is attained. The change in the decreasing rate of the infection is probability obtained at a value of approximately 0.29 when the population is completely immunized against tuberculosis, which results in a greater decreasing rate for control against HIV. Figure 5(c) shows the union of the infection probability of tuberculosis and HIV in the multilayer network. A decreasing rate of the infection probability increases at a value of approximately 0.22 due to the increase in the decreasing rate of tuberculosis and decreases at a value of 


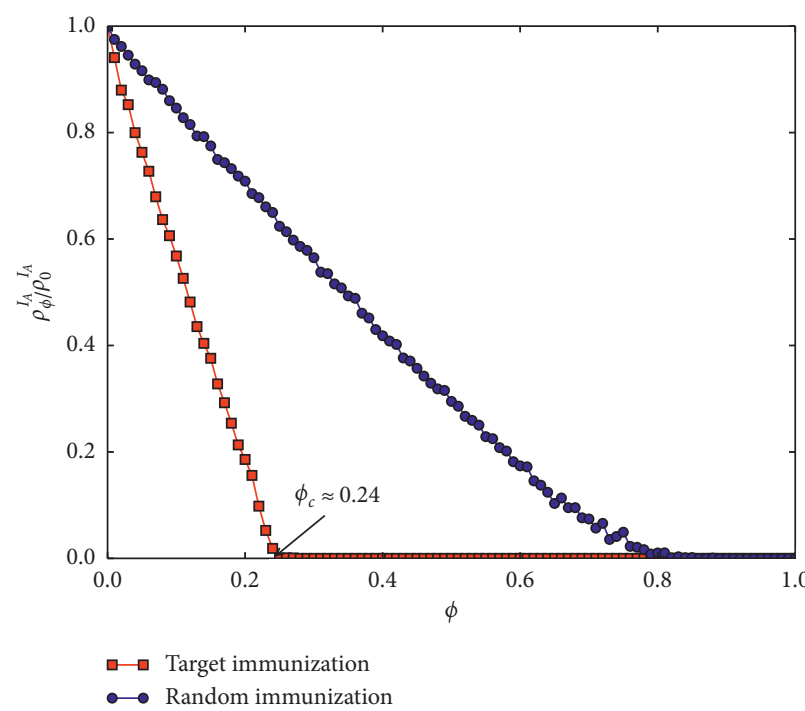

(a)

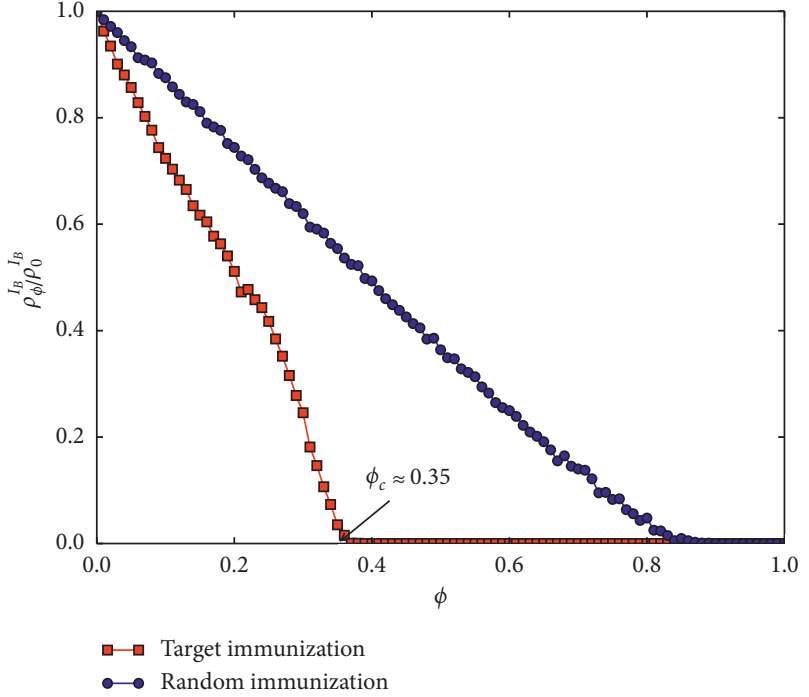

(b)

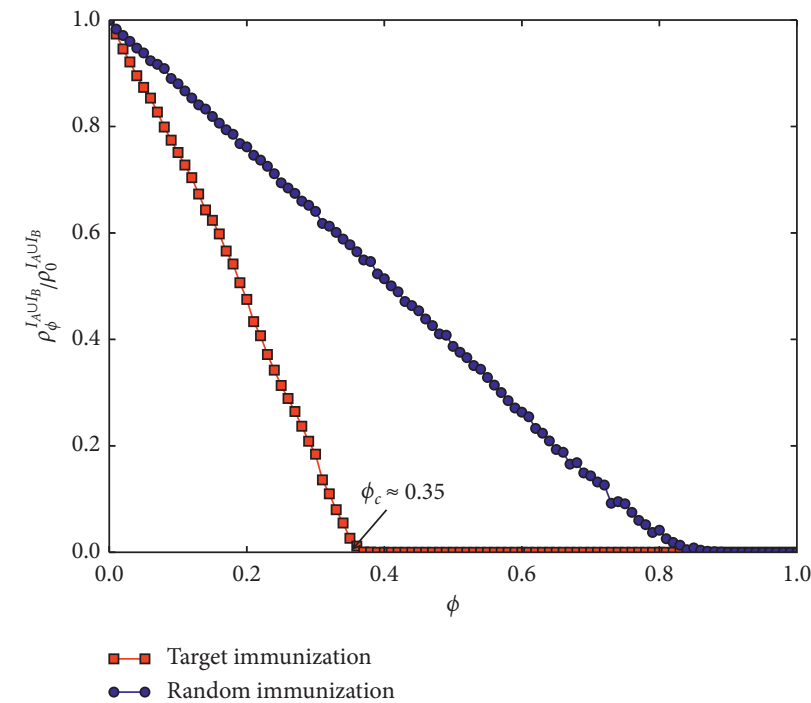

(c)

FIGURE 4: Relative size $\left(\rho_{\phi} / \rho_{0}\right)$ of infected clusters versus the immunization probability $\phi$ for multiplex target or random control in a doublelayer network that includes hepatitis B virus and hepatitis C virus: (a) hepatitis C virus-infected clusters, (b) hepatitis B virus-infected clusters, and (c) clusters infected with both hepatitis C and B viruses. The arrow denotes the immunization threshold $\phi_{c}$ of our novel Multiplex PageRank target control.

approximately 0.29 because only the HIV rate decreases. We find that our Multiplex PageRank target control strategy is capable of immunizing fewer nodes to achieve a greater effect on both viruses simultaneously in the multilayer network.

Because an individual who is infected with malaria or HIV will be more susceptible to the other virus and the degree of enhancement of HIV obtained with malaria infection is stronger than that of malaria with HIV [54], we adopt $c_{A}^{I_{B}}=1.8$ for malaria and $c_{B}^{I_{A}}=1.5$ for HIV. As there are more propagation routes for HIV than malaria, we adopt $A$ to denote the propagation routes of malaria and $B$ to denote the propagation routes of HIV. Based on the decision to mainly immunize against HIV and only sometimes immunize against malaria, we adopt the distribution coefficients $\beta_{A}=0.35$ and $\beta_{B}=0.5$ because HIV is more dangerous than malaria. The simulation results are shown in Figure 6. As shown in Figure 6(a), the immunization threshold of the multiplex random control of malaria is at a high value about 0.8 , and the decreasing rate of the curve is constant. We also find a change in the decreasing rate of the infection probability of multiplex target control of malaria before the immunization threshold is reached, which indicates that malaria is first enhanced by HIV. The change in the decreasing rate of the infection probability occurs at a value of approximately 0.25 when HIV has propagated to the top-ranked nodes, which are immunized, and this propagation results in an increase in the decreasing rate of the 


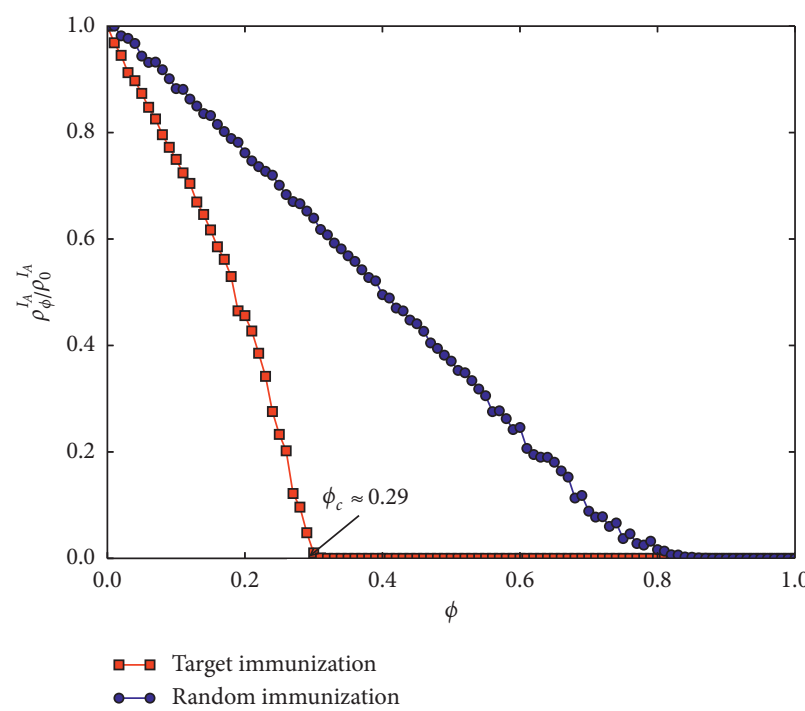

(a)

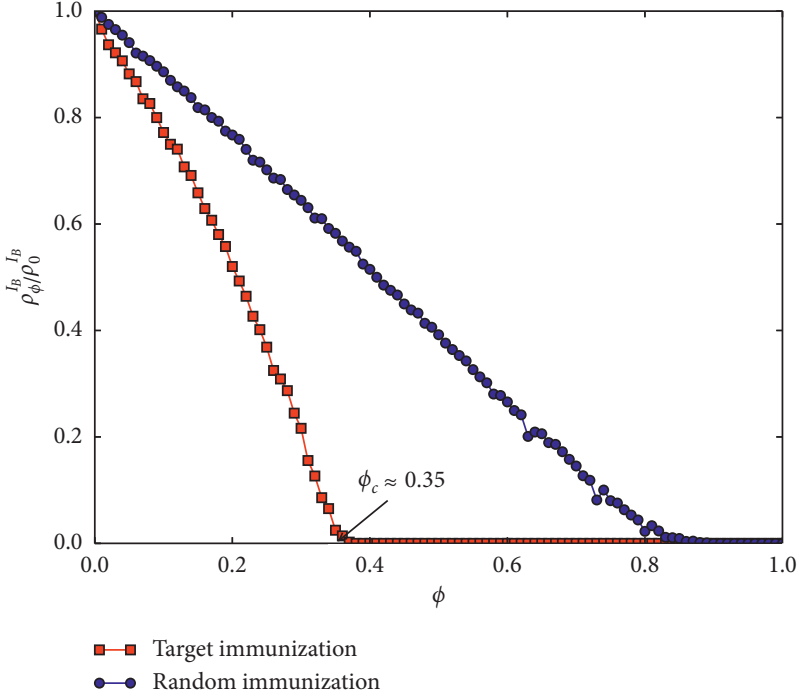

(b)

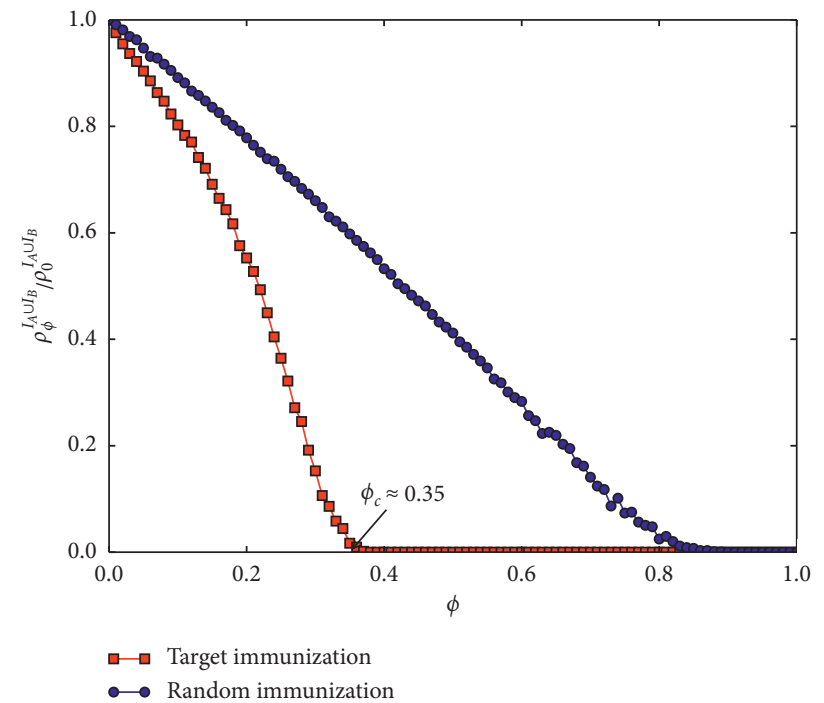

(c)

FIGURE 5: Relative size $\left(\rho_{\phi} / \rho_{0}\right)$ of infected clusters versus the immunization probability $\phi$ for multiplex target or random control in a doublelayer network that includes tuberculosis and HIV: (a) clusters infected with tuberculosis, (b) clusters infected with HIV, and (c) clusters infected with both tuberculosis $\phi_{c}$ of our novel Multiplex PageRank target control.

infection probability of malaria. However, even though the enhancement of HIV obtained with malaria is stronger than that of malaria achieved with HIV, malaria is constrained by its fewer propagation routes, and thus, the population is completely immunized against malaria before it is immunized against HIV. Moreover, as shown in Figure 6(b), the immunization threshold of the multiplex random control of $\mathrm{HIV}$ is at a high value about 0.8 , and the decreasing rate of the curve is constant. We also find some changes in the decreasing rate of the infection probability of multiplex target control of HIV before the immunization threshold is achieved, which indicates that malaria is first enhanced by $\mathrm{HIV}$. The first change in the decreasing rate of the infection probability occurs at a value of approximately 0.25 when malaria has propagated to the top-ranked nodes, which are mostly immunized, and this results in an increase in the decreasing rate of the HIV infection probability. The second change observed at a value of approximately 0.30 refers to an increase in the decreasing rate due to complete control against malaria, which leads HIV without any enhancement. Figure 6(c) presents the union of the infection probabilities of malaria and HIV in the multilayer network. The decreasing rate of the infection probability increases at a value of approximately 0.25 due to increases in both malaria and HIV and declines at a value of approximately 0.30 because only HIV infection remained. The results show that our Multiplex PageRank target control strategy immunizes fewer nodes to achieve a greater effect on both viruses simultaneously in the multilayer network.

Figures 2-6 show the relative sizes of the infected clusters versus the immunization probability in several actual cases. It is noted that the multiplex random control strategy in 


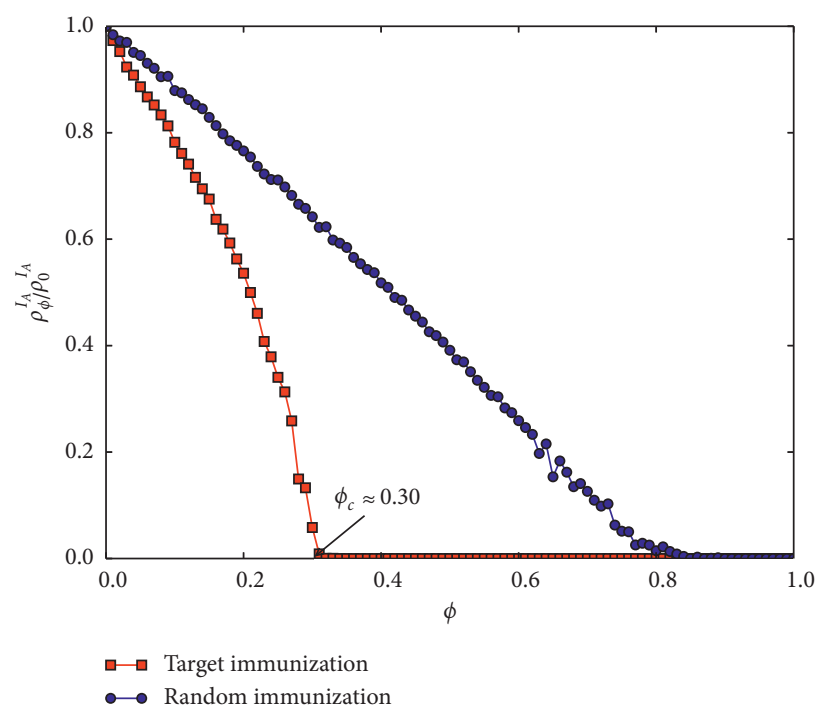

(a)

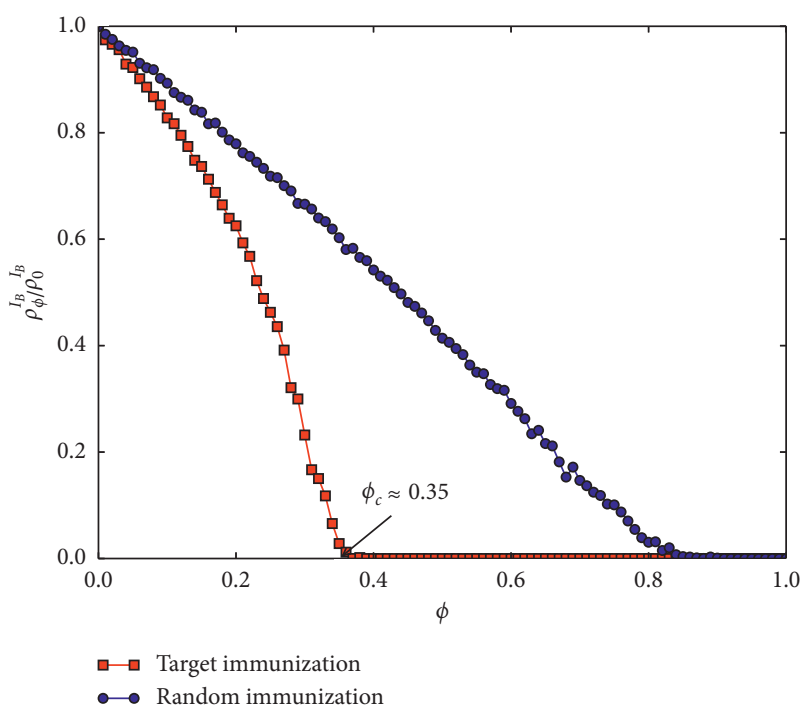

(b)

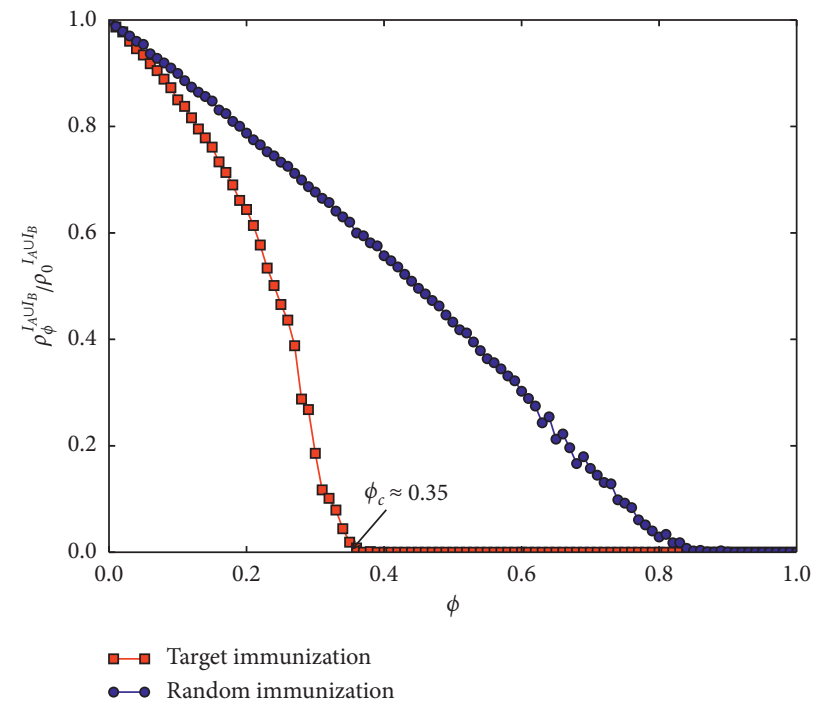

(c)

Figure 6: Relative size $\left(\rho_{\phi} / \rho_{0}\right)$ of infected clusters versus the immunization probability $\phi$ for multiplex target or random control in a doublelayer network including malaria and HIV: (a) malaria-infected clusters, (b) HIV-infected clusters, and (c) clusters infected with both malaria and HIV. The arrow denotes the immunization threshold $\phi_{\mathrm{c}}$ of our novel Multiplex PageRank target control.

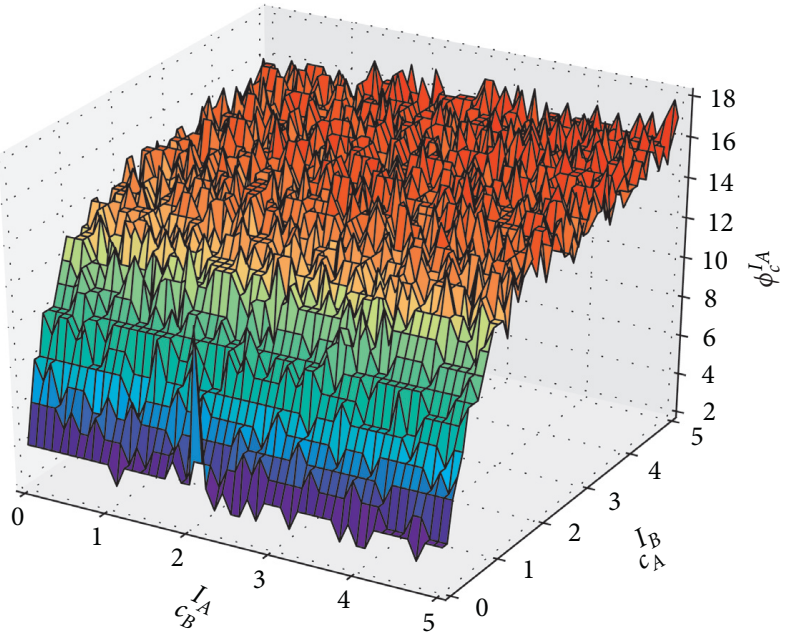

(a)

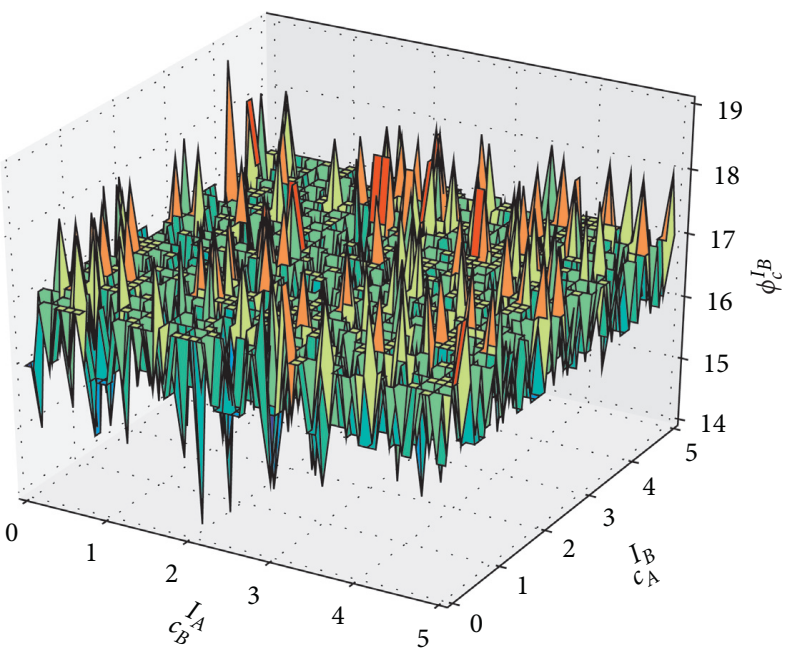

(b)

Figure 7: Continued. 


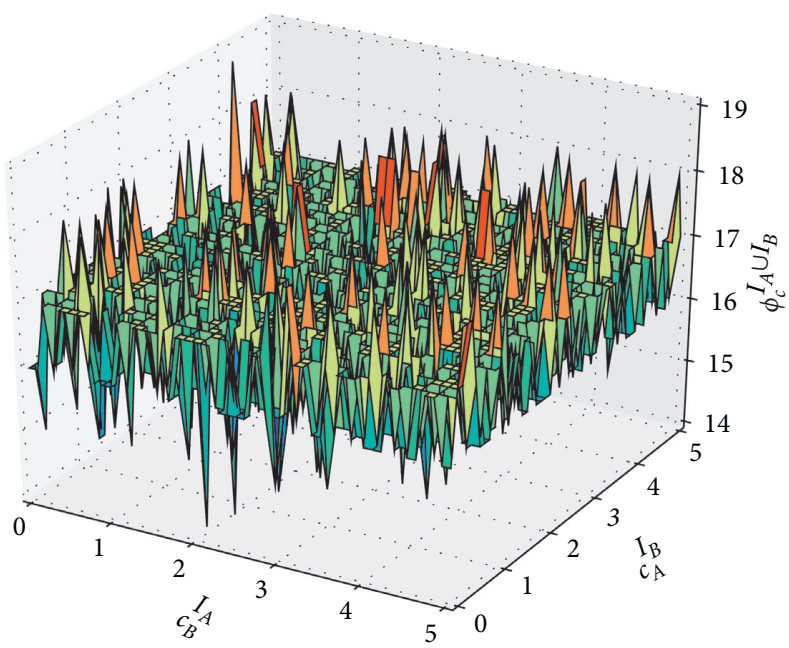

(c)

Figure 7: Novel Multiplex PageRank target immunization thresholds $\phi_{c}$ versus interaction coefficients of multiple-virus propagation. (a) Target immunization threshold of $I_{A}$. (b) Target immunization threshold of $I_{B}$. (c) Target immunization threshold of $I_{A} \cup I_{B}$.

these double-layer networks with different interaction coefficients has little difference. The results demonstrate the effectiveness of our multiplex target control strategy in multilayer networks. Furthermore, the figures present the immunization threshold $\phi_{c}$ of our novel Multiplex PageRank target control versus the interaction coefficients $c_{A}^{I_{B}}$ and $c_{B}^{I_{A}}$. By setting $\alpha_{A}^{S_{B}}=0.2$ and $\alpha_{B}^{S_{A}}=0.2$, both the interaction coefficients are limited from 0 to 5 because $\alpha_{A}^{I_{B}} \leq 1$ and $\alpha_{B}^{I_{A}} \leq 1$. Using 0.1 intervals for the interaction coefficients, we divide both of them into 51 partitions. The immunization probability $\phi$ ranges from 0 to 1 at a 0.01 interval, which results in the division of the probability into $101 \mathrm{com}$ partments. Adopting 100 time steps 1000 times for 50 networks with a size of 10,000 , the computer simulations take a long time. We exploit parallel computing, including multiple CPUs and multiple GPUs, to solve this problem, and the results are shown in Figure 7. As shown in Figure 7(a), the immunization threshold of the virus in $A$ is majorly impacted by the interaction coefficient $c_{A}^{I_{B}}$. The results shown in Figure 7(b) demonstrate that the immunization threshold of the virus in $B$ is impacted by the ratio of $c_{B}^{I_{A}}$ to $c_{A}^{I_{B}}$. Figure $7(\mathrm{c})$ reveals that the immunization threshold of the total infectious is decided by the virus in $B$ because it has more propagation routes.

\section{Conclusion}

This paper has presented a novel Multiplex PageRank target control strategy on multiple-virus interaction and propagation in multilayer networks compared with the multiplex random control. Using the proposed strategy, we have simulated several computer experiments that allow us to obtain the relative size of the steady probability versus the immunization probability and the critical immunization threshold for several actual cases. Furthermore, we have shown the critical immunization threshold of our novel Multiplex PageRank target control versus different interaction coefficients of our multiple-virus propagation, which is useful for further practical applications.

\section{Data Availability}

All the network data used in the paper are artificial network data, and the constructing measures are included with the supplementary information file.

\section{Conflicts of Interest}

The authors declare that they have no conflicts of interest.

\section{References}

[1] Z. Wang, L. Wang, and M. Perc, "Degree mixing in multilayer networks impedes the evolution of cooperation," Physical Review E, vol. 89, no. 5, Article ID 052813, 2014.

[2] Q. Jin, L. Wang, C.-Y. Xia, and Z. Wang, "Spontaneous symmetry breaking in interdependent networked game," Scientific Reports, vol. 4, no. 1, p. 4095, 2014.

[3] L. Wang, Y. Zhang, Z. Wang, and X. Li, "The impact of human location-specific contact pattern on the SIR epidemic transmission between populations," International Journal of Bifurcation and Chaos, vol. 23, no. 05, Article ID 1350095, 2013.

[4] L. Wang, Z. Wang, Y. Zhang, and X. Li, "How human location-specific contact patterns impact spatial transmission between populations?" Scientific Reports, vol. 3, no. 1, p. 1468, 2013.

[5] L. Wang, X. Li, Y.-Q. Zhang, Y. Zhang, and K. Zhang, "Evolution of scaling emergence in large-scale spatial epidemic spreading," PLoS ONE, vol. 6, no. 7, Article ID e21197, 2011.

[6] D. Zhao, L. Li, H. Peng, Q. Luo, and Y. Yang, "Multiple routes transmitted epidemics on multiplex networks," Physics Letters A, vol. 378, no. 10, pp. 770-776, 2014.

[7] B. Min, S. Do Yi, K.-M. Lee, and K.-I. Goh, "Network robustness of multiplex networks with interlayer degree correlations," Physical Review E, vol. 89, no. 4, Article ID 042811, 2014. 
[8] C. Buono, L. G. Alvarez-Zuzek, P. A. Macri, and L. A. Braunstein, "Epidemics in partially overlapped multiplex networks," PLoS ONE, vol. 9, no. 3, Article ID e92200, 2014.

[9] O. Yagan, D. Qian, J. Zhang, and D. Cochran, "Conjoining speeds up information diffusion in overlaying social-physical networks," IEEE Journal on Selected Areas in Communications, vol. 31, no. 6, pp. 1038-1048, 2013.

[10] J. Gómez-Gardenes, I. Reinares, A. Arenas, and L. M. Floría, "Evolution of cooperation in multiplex networks," Scientific Reports, vol. 2, no. 1, p. 620, 2012.

[11] J. Y. Kim and K.-I. Goh, "Coevolution and correlated multiplexity in multiplex networks," Physical Review Letters, vol. 111, no. 5, Article ID 058702, 2013.

[12] F. Battiston, V. Nicosia, and V. Latora, "Structural measures for multiplex networks," Physical Review E, vol. 89, no. 3, Article ID 032804, 2014.

[13] C. Granell, S. Gómez, and A. Arenas, "Dynamical interplay between awareness and epidemic spreading in multiplex networks," Physical Review Letters, vol. 111, no. 12, Article ID 128701, 2013.

[14] V. Nicosia, G. Bianconi, V. Latora, and M. Barthelemy, "Growing multiplex networks," Physical Review Letters, vol. 111, no. 5, Article ID 058701, 2013.

[15] S. Gomez, A. Diaz-Guilera, J. Gomez-Gardenes, C. J. PerezVicente, Y. Moreno, and A. Arenas, "Diffusion dynamics on multiplex networks," Physical Review Letters, vol. 110, no. 2, Article ID 028701, 2013.

[16] M. Salehi, R. Sharma, M. Marzolla, M. Magnani, P. Siyari, and D. Montesi, "Spreading processes in multilayer networks," IEEE Transactions on Network Science and Engineering, vol. 2, no. 2, pp. 65-83, 2015.

[17] S. Boccaletti, G. Bianconi, R. Criado et al., "The structure and dynamics of multilayer networks," Physics Reports, vol. 544, no. 1, pp. 1-122, 2014.

[18] F. Caccioli, M. Shrestha, C. Moore, and J. D. Farmer, "Stability analysis of financial contagion due to overlapping portfolios," Journal of Banking \& Finance, vol. 46, pp. 233-245, 2014.

[19] M. J. O. Pocock, D. M. Evans, and J. Memmott, "The robustness and restoration of a network of ecological networks," Science, vol. 335, no. 6071, pp. 973-977, 2012.

[20] J. Iacovacci and G. Bianconi, "Extracting information from multiplex networks," Chaos: An Interdisciplinary Journal of Nonlinear Science, vol. 26, no. 6, Article ID 065306, 2016.

[21] R. Gallotti and M. Barthelemy, "Anatomy and efficiency of urban multimodal mobility," Scientific Reports, vol. 4, no. 1, p. 6911, 2014.

[22] X. Wang and H. Su, "Completely model-free RL-based consensus of continuous-time multi-agent systems," Applied Mathematics and Computation, vol. 382, Article ID 125312, 2020.

[23] Y. Zhang, G. Wen, G. Chen et al., "Gaming temporal networks," IEEE Transactions on Circuits and Systems II: Express Briefs, vol. 66, no. 4, pp. 672-676, 2018.

[24] X. Wang, X. Wang, H. Su, and J. Lam, "Coordination control for uncertain networked systems using interval observers," IEEE Transactions on Cybernetics, 2019.

[25] B. Karrer and M. E. Newman, "Competing epidemics on complex networks," Physical Review E, vol. 84, no. 3, Article ID 036106, 2011.

[26] B. Min and K. Goh, "Layer-crossing overhead and information spreading in multiplex social networks," Seed, vol. 21, no. T22, p. T12, 2013.

[27] V. Marceau, P.-A. Noël, L. Hébert-Dufresne, A. Allard, and L. J. Dubé, "Modeling the dynamical interaction between epidemics on overlay networks," Physical Review E, vol. 84, no. 2, Article ID 026105, 2011.

[28] S. Funk and V. A. Jansen, "Interacting epidemics on overlay networks," Physical Review E, vol. 81, no. 3, Article ID 036118, 2010.

[29] M. E. Newman and C. R. Ferrario, "Interacting epidemics and coinfection on contact networks," PLOS ONE, vol. 8, no. 8, Article ID e71321, 2013.

[30] Y.-Y. Ahn, H. Jeong, N. Masuda, and J. D. Noh, "Epidemic dynamics of two species of interacting particles on scale-free networks," Physical Review E, vol. 74, no. 6, Article ID 066113, 2006.

[31] C. Gao and J. Liu, "Modeling and restraining mobile virus propagation," IEEE Transactions on Mobile Computing, vol. 12, no. 3, pp. 529-541, 2013.

[32] D. Zhao, H. Peng, L. Li, Y. Yang, and S. Li, "An efficient patch dissemination strategy for mobile networks," Mathematical Problems in Engineering, vol. 2013, pp. 1-13, 2013.

[33] D. Zhao, L. Li, S. Li, Y. Huo, and Y. Yang, "Identifying influential spreaders in interconnected networks," Physica Scripta, vol. 89, no. 1, Article ID 015203, 2013.

[34] C. Gao, J. Liu, and N. Zhong, "Network immunization with distributed autonomy-oriented entities," IEEE Transactions on Parallel and Distributed Systems, vol. 22, no. 7, pp. 1222-1229, 2011.

[35] B. Gao, Z. Deng, and D. Zhao, "Competing spreading processes and immunization in multiplex networks," Chaos, Solitons \& Fractals, vol. 93, pp. 175-181, 2016.

[36] L. G. A. Zuzek, C. Buono, and L. A. Braunstein, "Epidemic spreading and immunization strategy in multiplex networks," Journal of Physics: Conference Series, vol. 640, no. 1, Article ID 012007, 2015.

[37] R. Cohen, S. Havlin, and D. Ben-Avraham, "Efficient immunization strategies for computer networks and populations," Physical Review Letters, vol. 91, no. 24, Article ID 247901, 2003.

[38] L. K. Gallos, F. Liljeros, P. Argyrakis, A. Bunde, and S. Havlin, "Improving immunization strategies," Physical Review E, vol. 75, no. 4, Article ID 045104, 2007.

[39] Y. Chen, G. Paul, S. Havlin, F. Liljeros, and H. E. Stanley, "Finding a better immunization strategy," Physical Review Letters, vol. 101, no. 5, Article ID 058701, 2008.

[40] L. Wang, Y. Zhang, T. Huang, and X. Li, "Estimating the value of containment strategies in delaying the arrival time of an influenza pandemic: a case study of travel restriction and patient isolation," Physical Review E, vol. 86, no. 3, Article ID 032901, 2012.

[41] L. Wang and X. Li, "Spatial epidemiology of networked metapopulation: an overview," Chinese Science Bulletin, vol. 59, no. 28, pp. 3511-3522, 2014.

[42] H. Ishii, R. Tempo, and E.-W. Bai, "A web aggregation approach for distributed randomized PageRank algorithms," IEEE Transactions on Automatic Control, vol. 57, no. 11, pp. 2703-2717, 2012.

[43] L. Solá, M. Romance, R. Criado, J. Flores, A. García del Amo, and S. Boccaletti, "Eigenvector centrality of nodes in multiplex networks," Chaos: An Interdisciplinary Journal of Nonlinear Science, vol. 23, no. 3, Article ID 033131, 2013.

[44] M. D. Domenico, A. Solé-Ribalta, E. Omodei, S. Gómez, and A. Arenas, "Ranking in interconnected multilayer networks reveals versatile nodes," Nature Communications, vol. 6, no. 1, 2015.

[45] A. Solé-Ribalta, M. Domenico, S. Gómez, and A. Arenas, "Centrality rankings in multiplex networks," in Proceedings of 
the 2014 ACM conference on Web science, vol. 9, Bloomington, Indiana, June 2014.

[46] F. Pedroche, M. Romance, and R. Criado, "A biplex approach to PageRank centrality: from classic to multiplex networks," Chaos, vol. 26, no. 6, 2016.

[47] B. Jiang, K. Kloster, D. F. Gleich, and M. Gribskov, “AptRank: an adaptive PageRank model for protein function prediction on Bi-relational graphs," Bioinformatics, vol. 33, no. 12, 2016.

[48] J. Iacovacci, C. Rahmede, A. Arenas, and G. Bianconi, "Functional multiplex PageRank," Europhysics Letters, vol. 116, no. 2, 2016.

[49] A. Halu, R. J. Mondragón, P. Panzarasa, and G. Bianconi, "Multiplex pagerank," PLoS ONE, vol. 8, no. 10, Article ID e78293, 2013.

[50] H. Emerson, "Measles and whooping cough," American Journal of Public Health and the Nations Health, vol. 27, no. 6 Suppl, pp. 1-58, 1937.

[51] L.-C. Chen and K. M. Carley, "The impact of countermeasure propagation on the prevalence of computer viruses," IEEE Transactions on Systems, Man and Cybernetics, Part B (Cybernetics), vol. 34, no. 2, pp. 823-833, 2004.

[52] A. Alberti et al., "The interaction between hepatitis B virus and hepatitis $\mathrm{C}$ virus in acute and chronic liver disease," Journal of Hepatology, vol. 22, no. 1 Suppl, pp. 38-41, 1995.

[53] E. L. Corbett, C. J. Watt, N. Walker et al., "The growing burden of tuberculosis," Archives of Internal Medicine, vol. 163, no. 9, pp. 1009-1021, 2003.

[54] A. Alemu, Y. Shiferaw, Z. Addis, B. Mathewos, and W. Birhan, "Effect of malaria on HIV/AIDS transmission and progression," Parasites \& Vectors, vol. 6, no. 1, p. 18, 2013. 